\title{
Editorial
}

Interational Axchives of
Allergy
Immunology

DOI: $10.1159 / 000436971$

Published online: August 12, 2015

\section{Peri-Operative Anaphylaxis: Beyond Drugs and Latex}

\author{
Mamidipudi Thirumala Krishna ${ }^{a}$ b Aarnoud Huissoon $^{a}$ \\ ${ }^{a}$ Department of Allergy and Immunology, Birmingham Heartlands Hospital, Heart of England NHS Foundation \\ Trust, and ${ }^{\mathrm{b}}$ School of Life and Health Sciences, Aston University, Birmingham, UK
}

Chlorhexidine gluconate is an antiseptic that has been used worldwide in clinical practice since 1954. It is available as a $0.5-4.0 \%$ solution and has a broad spectrum of activity against Gram-positive and Gram-negative bacteria, facultative anaerobes, yeasts and HIV [1]. It is employed to decontaminate people and medical devices. Healthcare-associated infections are the most common adverse events occurring in hospitalised patients and are reported in up to $10 \%$ of inpatients in acute-care hospitals [2]. Hand-washing with chlorhexidine is an effective means of eliminating skin flora by $86-92 \%$, and is crucial for combatting and reducing the incidence of methicillinresistant Staphylococcus aureus (MRSA) infection [1,3,4].

The counter argument is that increased rates of exposure to chlorhexidine have probably contributed to greater sensitisation and the emergence of IgE-mediated anaphylaxis, which is sometimes near-fatal, particularly in the context of surgical procedures. The first case of chlorhexidine-induced allergic shock was reported in the Japanese literature by Takeda et al. [5] in 1985. A PubMed search using the key words 'chlorhexidine' and 'anaphylaxis' showed 97 hits in the period 1974-2015, with 2, 15, 37 and 43 publications in 1974-1984, 1985-1994, 1995-2005 and 2006-2015, respectively, mostly in the form of case reports or series. These publications prompted the US Food and Drug Administration (FDA) to issue an alert to the medical fraternity about the possibility of serious anaphylactic reactions to chlorhexidine-impregnated medical devices [6]. The literature on chlorhexidine allergy compares the emergence of the sensitisation and allergy to chlorhexidine to the latex allergy epidemic that was feared in the (c) 2015 S. Karger AG, Base

$1018-2438 / 15 / 1672-0101 \$ 39.50 / 0$ 1990s [7-9]. We and other study groups have shown that a significant proportion (5-10\%) of IgE-mediated anaphylaxis occurring during general anaesthesia is triggered by chlorhexidine $[8,10,11]$. To put this into perspective, the incidence rate of peri-operative (all causes) anaphylaxis during anaesthesia has been estimated to be 1 in 10,000-20,000 in France and Australia [12, 13].

Hong et al. [14] recently reported an interesting case in International Archives of Allergy and Immunology. This concerned recurrent anaphylaxis triggered by chlorhexidine used for skin and wound cleansing in a critically ill patient with necrotising fasciitis, septic shock and acute renal impairment on a background of hypertension, type 2 diabetes mellitus and coronary artery disease. It is not clear if the patient developed anaphylaxis after the first surgery because this may have been masked by underlying sepsis and was managed with intravenous fluids and inotropic support. However, the subsequent episodes, in particular the third and fourth, when his general condition had improved significantly, manifested as acute hypotension, bronchospasm and flushing peri-operatively, which meet the World Allergy Organisation (WAO) diagnostic criteria for anaphylaxis [15]. However, anaphylaxis was not suspected until after the fourth episode, and was confirmed by a modest elevation in acute serum tryptase (16.7 $\mu \mathrm{g} / \mathrm{l})$. Interestingly, the patient developed Kounis syndrome, a likely consequence of coronary hypoperfusion, thus highlighting the importance of prompt recognition and treatment with adrenaline to reverse hypotension.

This case can be used to illustrate some 'clinical pearls' for anaesthetists and allergists. First, anaphylaxis should

\section{KARGER 125}

E-Mail karger@karger.com

www.karger.com/iaa
Correspondence to: Dr. M.T. Krishna

Department of Allergy and Immunology, Birmingham Heartlands Hospital Bordesley Green East

Birmingham B9 5SS (UK)

E-Mail mtkrishna@yahoo.com 
be included as a differential diagnosis for acute hypotension or cardiopulmonary arrest that occurs during general anaesthesia. Second, serial serum tryptase measurements (including baseline at $\geq 24 \mathrm{~h}$ ) should be undertaken in such clinical scenarios. We recently reported the sensitivity, specificity, positive predictive value and negative predictive value of an acute serum tryptase level $>15.7$ $\mu \mathrm{g} / \mathrm{l}$ to be 75, 68.4, 82 and 59\%, respectively, for IgE-mediated anaphylaxis during general anaesthesia [8]. Baseline serum tryptase is a useful surrogate biomarker for disorders of mast-cell overload. Third, chlorhexidine should be considered as a possible culprit in all cases of suspected anaphylaxis during general anaesthesia, particularly when there is no immediate temporal association with the administration of induction agents and when the reaction occurs towards the middle or end of the procedure or in the immediate postoperative phase [16]. Serious anaphylaxis to chlorhexidine-impregnated central venous catheters has been reported within seconds following insertion [17]. Scrutiny of the chronology, timing and route of exposure to drugs administered during general anaesthesia, information regarding the antiseptic used in prepping skin, whether or not a central venous catheter was used and the details of urinary catheterisation, including the lubricant employed, should be obtained. The Association of Anaesthetists of Great Britain and Ireland has produced a proforma (www.aagbi.org/ sites/default/files/anaphylaxis_referralform_v2.doc) to help collate these details in a systematic manner in order to facilitate analysis and plan allergy tests. Fourth, providing the patient with a medical-alert bracelet or something similar as well as clear documentation of the allergy in their clinical records is essential, in order to avoid accidental re-exposure. Clear labelling of all medical devices such as central venous catheters, indicating that they are impregnated with chlorhexidine, is paramount for the enhancement of safety.

At the present time, the benefits of using chlorhexidine for its antiseptic and disinfectant properties in the clinical environment outweigh the relatively rare but real risk of anaphylaxis. The prevalence of sensitisation to this agent in the general population and among healthcare workers is not known; such data would be invaluable to be able to ascertain if there is, indeed, a global chlorhexidine allergy epidemic. Based on current evidence, this seems unlikely, especially considering the widespread use of chlorhexidine and the apparently low rate of the resulting minor or severe allergic reactions. It is possible that such reactions are not recognised for what they are and are therefore underreported. In this event, strategies to limit exposure in the clinical environment may be necessary, despite the crucial role for chlorhexidine in reducing healthcare-associated infections. In the meantime, efforts should focus on raising the awareness of the diagnostic possibility of anaphylaxis, and protecting patients with known chlorhexidine allergy from accidental re-exposure which is potentially catastrophic.

\section{References}

$\checkmark 1$ Milstone AM, Passaretti CL, Perl TM: Chlorhexidine: expanding the armamentarium for infection control and prevention. Clin Infect Dis 2008;46:274-281.

$>2$ Burke JP: Infection control - a problem for patient safety. N Engl J Med 2003;348:651656.

$>3$ Lowbury EJ, Lilly HA: Use of 4 per cent chlorhexidine detergent solution (Hibiscrub) and other methods of skin disinfection. $\mathrm{Br}$ Med J 1973;1:510-515.

4 Askgaard K: Effect of some antiseptic detergents for surgical hand washing. Ugeskr Laeger 1975;137:2515-2518.

$>5$ Takeda K, Inoue K, Matsuya T, et al: Allergic shock possibly induced by chlorhexidine: report of a case. Osaka Daigaku Shigaku Zasshi 1985;30:221-225.

6 Center for Devices and Radiological Health US FDA: FDA Public Health Notice: potential hypersensitivity reactions to chlorhexidine impregnated medical devices, March 1998.
7 Nagendran V, Wicking J, Ekbote A, Onyekwe T, Garvey LH: IgE-mediated chlorhexidine allergy: a new occupational hazard? Occup Med (Lond) 2009;59:270-272.

$>8$ Krishna MT, York M, Chin T, et al: Multicentre retrospective analysis of anaphylaxis during general anaesthesia in the United Kingdom: aetiology and diagnostic performance of acute serum tryptase. Clin Exp Im munol 2014;178:399-404.

$>9$ Rutkowski K, Wagner A: Chlorhexidine: a new latex? Eur Urol 2015, Epub ahead of print.

-10 Opstrup MS, Malling HJ, Kroigaard M, Mosbech H, Skov PS, Poulsen LK, et al: Standardized testing with chlorhexidine in perioperative allergy - a large single-centre evaluation. Allergy 2014;69:1390-1396.

11 Antunes J, Kochuyt AM, Ceuppens JL: Perioperative allergic reactions: experience in a Flemish referral centre. Allergol Immunopathol (Madr) 2014;42:348-354.

12 Laxenaire MC: Epidemiology of anesthetic anaphylactoid reactions. Fourth multicenter survey (July 1994-December 1996). Ann Fr Anesth Reanim 1999;18:796-809.
13 Fisher MM, Baldo BA: The incidence and clinical features of anaphylactic reactions during anesthesia in Australia. Ann Fr Anesth Reanim 1993;12:97-104.

14 Hong CC, Wang SM, Nather A, Tan JHJ, Tay $\mathrm{SH}$, Poon KH: Chlorhexidine anaphylaxis masquerading as septic shock. Int Arch Allergy Immunol 2015;167:16-20.

-15 Simons FE, Ardusso LR, Bilo MB, El-Gamal YM, Ledford DK, Ring J, et al: World Allergy Organization anaphylaxis guidelines: summary. J Allergy Clin Immunol 2011;127:587593 e1-22.

-16 Parkes AW, Harper N, Herwadkar A, Pumphrey R: Anaphylaxis to the chlorhexidine component of Instillagel: a case series. $\mathrm{Br} \mathrm{J}$ Anaesth 2009;102:65-68.

-17 Jee R, Nel L, Gnanakumaran G, Williams A, Eren E: Four cases of anaphylaxis to chlorhexidine impregnated central venous catheters: a case cluster or the tip of the iceberg? Br J Anaesth 2009; 103:614-615. 